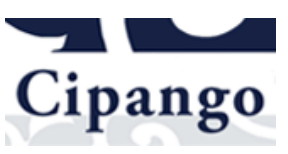

\title{
Cipango
}

Cahiers d'études japonaises

16 | 2009

L'invention des « arts populaires » - Yanagi Sōetsu et le Mingei

\section{À la poursuite infinie des désirs intérieurs : Yanagi Sōetsu avant le Mingei}

The endless pursuit of inner desires: Yanagi Sōetsu before Mingei

\section{Michael Lucken}

\section{(2) OpenEdition}

\section{Journals}

Édition électronique

URL : https://journals.openedition.org/cipango/388

DOI : $10.4000 /$ cipango.388

ISSN : 2260-7706

Éditeur

INALCO

Édition imprimée

Date de publication : 1 janvier 2009

Pagination : 23-41

ISSN : $1164-5857$

Référence électronique

Michael Lucken, « À la poursuite infinie des désirs intérieurs : Yanagi Sōetsu avant le Mingei », Cipango [En ligne], 16 | 2009, mis en ligne le 15 novembre 2011, consulté le 30 juin 2021. URL : http:// journals.openedition.org/cipango/388; DOI : https://doi.org/10.4000/cipango.388

Ce document a été généré automatiquement le 30 juin 2021

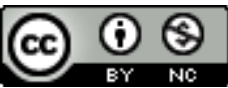

Cipango est mis à disposition selon les termes de la Licence Creative Commons Attribution - Pas d'Utilisation Commerciale 4.0 International. 


\title{
À la poursuite infinie des désirs intérieurs : Yanagi Sōetsu avant le Mingei
}

The endless pursuit of inner desires: Yanagi Sōetsu before Mingei

\author{
Michael Lucken
}

1 Vu du Japon, l'écrivain et critique Yanagi Sōetsu 柳宗悦 (1889-1961) apparait comme ayant eu deux vies : une première, qui couvre les années 1910 et qui correspond à son engagement au sein de la revue Shirakaba 白樺 (1910-1923) au cours de laquelle son intérêt s'est porté sur les mystiques chrétiens et certains artistes occidentaux comme Rodin ou Blake; une seconde, qui a débuté autour de 1920 et qui le vit s'engager en faveur du développement culturel de la Corée et d'une redécouverte ou d'une réinvention des arts populaires (mingei 民藝). Ces deux faces sont souvent opposées, néanmoins on a conscience que le mouvement des Arts populaires se situe dans le prolongement de l'extraordinaire aventure humaine et intellectuelle que constitua la publication de la revue Shirakaba.

2 En France, si le nom de Yanagi est un tant soit peu connu, c'est uniquement pour son implication dans le mouvement des Arts populaires, grâce notamment aux publications d'Élisabeth Frolet ou aux livres de Bernard Leach, dont une adaptation d'un texte de Yanagi, qui mettent l'accent sur cette partie de son œuvre ${ }^{1}$. En revanche, il n'y a en français aucune publication solide sur Shirakaba et l'on perçoit mal l'importance du rôle de cette revue ${ }^{2}$. L'exposition "L'esprit mingei au Japon » qui s'est tenue à l'automne 2008 au Musée du quai Branly est le reflet de cette méconnaissance. Mettant directement en lien des objets populaires anciens et du mobilier contemporain sans prendre le temps de montrer le contexte intellectuel et artistique de l'époque, elle tend à présenter l'action de Yanagi comme celle d'un esthète sans histoire qui aurait permis au design contemporain de retrouver ses racines dans la profondeur de la terre japonaise et extrême-orientale.

3 Je voudrais dans ce court article esquisser certains des enjeux de la pensée de Yanagi au cours des années 1910, d'une part pour mettre en évidence ses dynamiques, d'autre 
part, pour faire comprendre le cheminement qui a mené à l'émergence de sa conception originale des arts populaires, autrement dit pour replacer le Mingei dans une histoire. Je n'aborderai toutefois pas les liens qui l'unissent aux autres mouvements folkloristes à travers le monde, comme le Arts \& Crafts de William Morris, renvoyant pour cela aux travaux cités en note ${ }^{3}$.

4 Pour traiter ce sujet, j'examinerai en quatre temps deux questions centrales dans les textes rédigés par Yanagi au cours de cette période, à savoir celle de l'individu créateur et celle de l'œuvre d'art. La première permettra de montrer comment la conception du peuple, du collectif, du "nous ", présente de façon essentielle au sein du mouvement des Arts populaires, est la conséquence d'une crise "collective » de l'idéal du moi créateur qui animait les écrivains et les artistes au cours des années 1910. La seconde nous amènera à considérer la naissance du mouvement Mingei dans le prolongement d'un effort de réenchantement des images et du monde.

\section{L'individu}

Shirakaba est une revue mensuelle qui fut publiée d'avril 1910 à août 1923. Elle fut fondée par un groupe d'écrivains issus pour la plupart de l'École des Pairs (Gakushūin), dont les plus connus sont, outre Yanagi, Mushanokōji Saneatsu 武者小路実篤 (1885-1976), Shiga Naoya 志賀直哉 (1883-1971), Kinoshita Rigen 木下利立 (1886-1925) et les frères Arishima : Arishima Takeo 有島武郎 (1878-1923), Arishima Ikuma 有島生 馬 (1882-1974) et Satomi Ton 里見弶 (1888-1983). On parle de mouvement Shirakaba (Shirakaba-ha 白樺派) pour désigner au sens large l'ensemble des collaborateurs de la revue, voire cette génération tout entière.

De caractère à la fois ouvert et exigeant, Yanagi a largement contribué au rayonnement de la revue. Il a notamment su s'assurer la collaboration de personnalités diverses et brillantes, comme le romancier Nagayo Yoshirō 長與善郎 ${ }^{4}$ ou le dessinateur et potier Bernard Leach qui illustra la couverture de nombreux numéros ${ }^{5}$. Plus largement, grâce à une activité de lecture extrêmement soutenue des publications occidentales, il a dès les années 1910 influé sur toute une génération d'écrivains, d'intellectuels et d'artistes. Même si Mushanokōji Saneatsu, du fait de la force de sa parole et de son écriture, a tenu jusqu'en 1918 et la création de son Nouveau village (Atarashiki mura) ${ }^{6}$ une position dominante au sein du groupe, Yanagi en a été à la fois la cheville ouvrière et le moteur critique. 
Le groupe Shirakaba, 1912

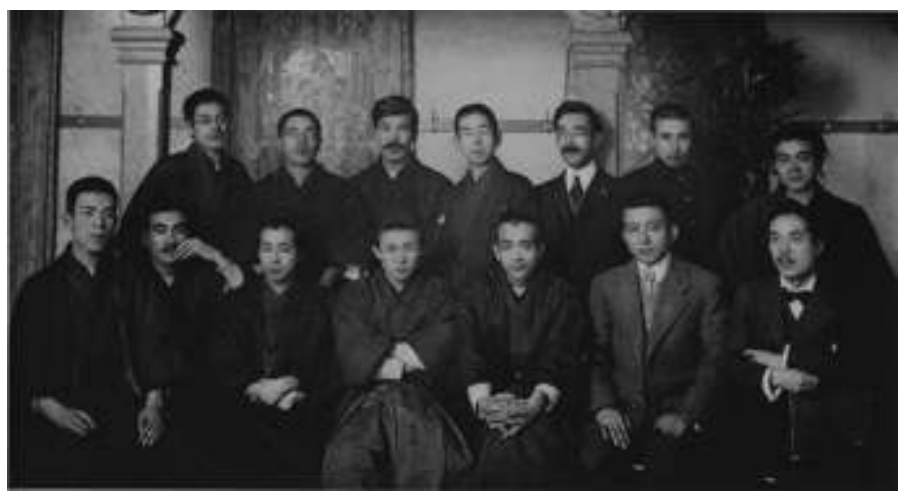

RÉUNION DU NOUVEL AN DE SHIRAKABA. KANDA, TŌKYō, 4 JANVIER 1912. DEBOUT, DE gAUCHE À DROITE : MUSHANOKŌJI SANEATSU, KOIZUMI MAgANE, TAKAMURA KŌTARō, KONOSHITA RIgEN, ŌgIMACHI KINKAZU, NAgAYO YOSHIRō, KUSAKA JIN (ŌgIMACHI SANEYOSHI). ASSIS, DE GAUCHE À DROITE : TANAKA USON, SHIgA NAOYA, SATOMI TON, YANAgI SŌETSU, SONOIKE KIN.YUKI, AOKI NAOSUKE, ARISHIMA IKUMA.

Le groupe Shirakaba, 1919

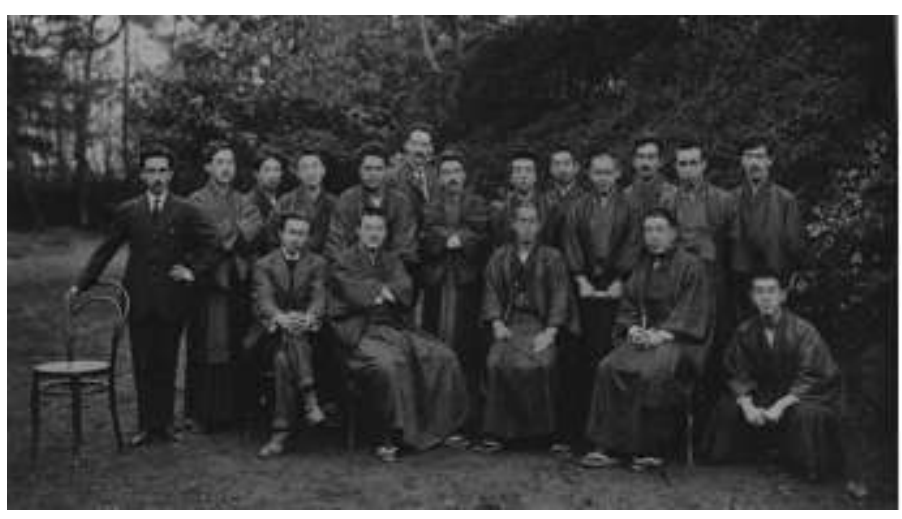

PHotographie Commémorative des 10 ans de shirakaba. PARC de SHIBA, tōkyō, 5 AVRIL 1919 DEBOUT, DE GAUCHE À DROITE : OZAKI KIHACHI, SATAKE HIROYUKI, YAWATA SEKITARō, SHINJō WAICHI, TSUBAKI SADAO, BERNARD LEACH, KOIZUMI MAgANE, KONDŌ KEIICHI, KINOSHITA RIgEN, KISHIDA RYŪSEI, SHIgA NAOYA, NAgAYO YOSHIRō, TAKAMURA KōTARō.

ASSIS, DE GAUCHE À DROITE : YANAgI SŌETSU, KIMURA SHŌHACHI, MUSHANOKŌJI SANEATSU, SEIMIYA HITOSHI, INUKAI TAKERU 
Couverture de la revue Shirakaba, avril 1910

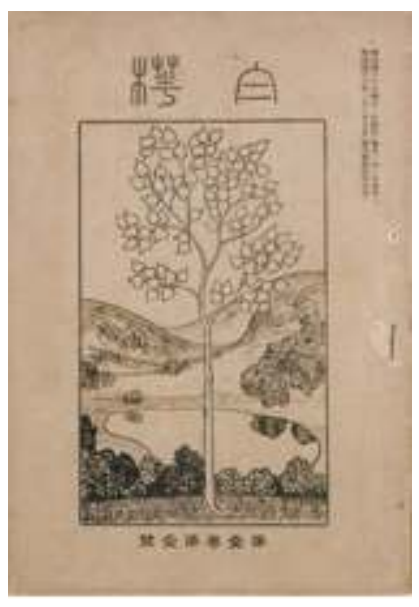

SHIRAKABA, N 1 -1, AVRIL 1910. ILLUSTRATION DE COUVERTURE : KOJIMA KIKUO.

Couverture de la revue Shirakaba, novembre 1910

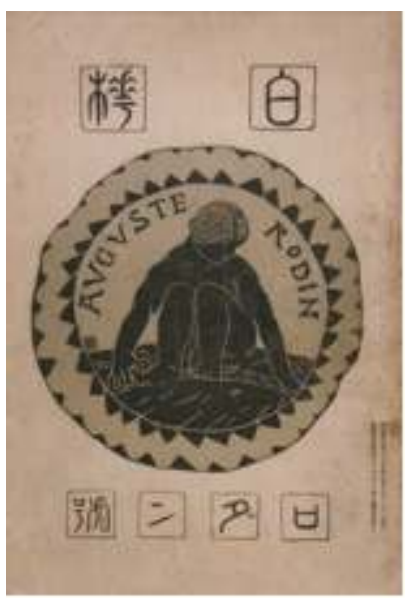

SHIRAKABA, N ${ }^{\circ}$ 1-8, NOVEMBRE 1910. ILLUSTRATION : MINAMI KUNZ̄̄.

Couverture de la revue Shirakaba, février 1918

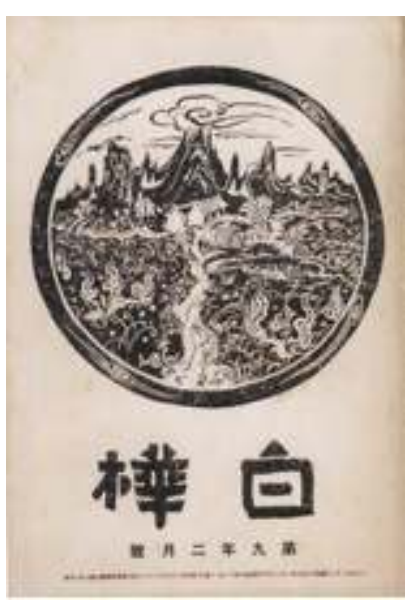

SHIRAKABA, N 9-2, FÉVRIER 1918. ILLUSTRATION DE COUVERTURE : BERNARD LEACH. 
Couverture de la revue Shirakaba, juillet 1918

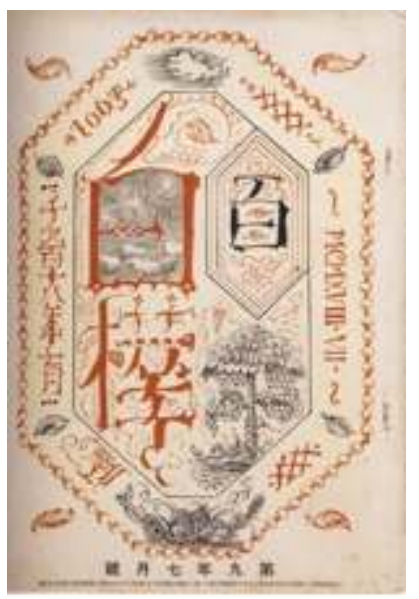

SHIRAKABA, N 9-7, JUILLET 1918. ILLUSTRATION : KISHIDA RYŪSEI.

7 Les premiers numéros de Shirakaba, entre 1910 et 1912, ont joué un rôle déterminant pour la réception au Japon de plusieurs artistes occidentaux majeurs, volontiers rassemblés à partir de 1910 et de l'exposition de Roger Fry à Londres sous l'étiquette du 《post-impressionnisme "( kōki inshō-ha 後期印象派 ou kō-inshō-ha 後印象派) ${ }^{7}$. On retient notamment le numéro sur Rodin publié en novembre 1910, ainsi que ceux sur Renoir et Van Gogh, respectivement en mars et octobre 1911. À chaque fois, Yanagi a pris la plume et s'est impliqué activement.

8 Toutefois, comme le remarque Kinoshita Nagahiro 木下長宏, c'est davantage la personnalité des artistes que leur œuvre qui a attiré l'attention ${ }^{8}$. Le caractère pathétique de la vie de Van Gogh, dont témoigne entre autres sa correspondance, a été à cette époque un élément essentiel de la reconnaissance du peintre hollandais au Japon. Cet aspect est tout à fait saillant dans " Les peintres de la révolution » (" Kakumei no gaka » 革命の画家), un article important de janvier 1912 que Yanagi publie sur Van Gogh, Gauguin, Cézanne et Matisse. Revenant en conclusion sur le rôle de l'individualité dans le processus de création, Yanagi écrit ${ }^{9}$ :

L'existence solennelle de l'individu est une réalité primordiale dont nous ne démordrons pas. Il n'y a aucun sens à l'existence en dehors d'une vie pour soi. Cultiver notre individualité est donc le seul et unique devoir qui nous incombe. Notre existence est le temps qui nous est imparti pour parfaire notre individualité. La vie humaine n'est rien d'autre que la poursuite infinie de nos désirs intérieurs. Ce n'est que lorsque nous donnons vie à nos désirs intérieurs que nous pouvons découvrir la valeur de l'existence. Van Gogh était précisément " un homme qui donnait de la vie ". Seule une telle façon de vivre peut positiver l'existence humaine. Et une vie positive est pure énergie. Elle a en elle une puissance et une autorité infinies. Enrichir nos existences, c'est donner corps à ce principe d'autorité. S'élever à travers soi-même met en action des forces universelles. Des individus supérieurs entraînent une humanité supérieure.

個性の厳肃なる存在は吾等の動かし難き第一義の事実である。此自己の生命を おいて吾等が存在には何の意義もない。かくて個性の充実とは吾等に与へられ たる唯一の本務である。吾等が生涯とは此個性の充全の為に賦与せられたる時 間である。人生とは此内部の要求に対する無限なる追求に外ならない。吾等は 此内心の要求に於て活ける時、始めて生の価值を知り得るのである。ゴオホは 斯くの如くにして「活ける人」であつた。活ける生命に於てのみ人生は其存在 を肯定し得るのである。然も肯定せられたる人生は力である。そこには無限の 威力と権威とがある。吾等が生命の充実とは此権威の体現である。個性にして 
偉大なる時、そは普遍の力を齊らさねば止まない。偉大なる個性の価値は引い

て偉大なる人類の価值である。

9 La mise en avant de l'« individu » (kosei 個性), de l'« ego » (jiko 自己) comme principe fondamental de la création est caractéristique des premiers textes de Yanagi. Elle a trouvé à s'alimenter à de multiples sources, notamment chez Nietzsche dont le chefd'œuvre Ainsi parlait Zarathoustra venait d'être rendu public dans une traduction d'Ikuta Chōkō 生田長江 ${ }^{10}$. Elle se retrouve chez Mushanokōji dans les articles qu'il écrit pour la rubrique 《Notes en corps six »(Rokugō zakkan 六号䧱感), la tribune libre de Shirakaba, ou chez le peintre Kishida Ryūsei 岸田劉生 (1891-1929) dont les liens avec la revue ont été étroits ${ }^{11}$.

10 Dans un article que ce dernier publie fin 1912 dans le Yomiuri shinbun à l'occasion de l'exposition de la Société du Fusain ${ }^{12}$, on peut lire ${ }^{13}$ :

En vérité, personne ne me comprendra, personne ne m'aidera aussi bien que je le fais moimême. Il est pénible de se regarder froidement. Mais la vacuité qu'il y a à vivre sans réfléchir à soi-même est insupportable. Pour moi, tenter de grandir passe forcément par la peinture. C'est pourquoi j'y plonge mon regard avec acuité. J'y plonge mon regard jusqu'à y percer un trou. Mes tableaux me dévoilent froidement tel que je suis, brisant toutes les illusions que j'ai à l'égard de moi-même. J'expose telle quelle ma réalité, je la scrute avec attention, mais, au sein de la désillusion, il ne se produit pas ce genre de révélation qui vous illumine le visage au quotidien.

実際、自己を一番よく理解し、一番よく自分に力をそへる者は自分自身を措 いて外に無い。自分は自分自身を、冷かに見つめる事を苦痛に思京。しかし、 自分自身の事を考えずに暮す空虚には堪へられない。自分が成長しようとする のはどうしても絵を描く事である。そして、それをお゙つと見つめる事である。 穴のあく程見つめる事である。自分の絵は、自分が自分に対する一切の幻を打 ち破つて冷かなありのままの自分を曝露して居る。自分は、自分の現実を只あ りのままに曝露して、それをまざまざと打ち眺めてその悲哀に日々笑む程に悟 りは開いて居ない。

11 Il n'y a aucun écart de fond entre cette déclaration et les propos de Yanagi. Même la langue est similaire, notamment le rythme des phrases qui est caractéristique des écrivains de cette génération. L'accent mis sur la puissance créatrice de l'«individu » est l'affaire de tout un groupe de jeunes gens qui cherchent dans l'écriture ou la peinture un moyen de l'exprimer.

Deux ans plus tard, Yanagi s'intéresse un peu moins à Van Gogh et Rodin. L'essentiel de son attention s'est déplacé vers l'artiste et poète anglais de la fin du XVIII et du début $\mathrm{XIX}^{\mathrm{e}}$ siècle, William Blake. L'œuvre de Blake est évidemment très différente de celle de Van Gogh ou de Renoir, mais les aspects formels intéressent peu Yanagi qui est surtout fasciné par l'attitude du graveur anglais, sa manière de se poser face au monde ${ }^{14}$ :

[Blake] détestait les contraintes et la censure. Il aspirait à ce que son pays soit un monde de liberté et de création. Il avait la certitude que les désirs intérieurs de l'homme recèlent une infinie puissance. Il prônait l'émanation infinie de l'homme à travers une réaction vive à l'égard de l'oppression. Son ambition immense de se réaliser lui-même est un idéal que, de toute sa vie, il n'a jamais perdu. Au nom de la vie, il a brisé, avec courage, toutes les règles établies. La liberté et l'affranchissement sont les portes uniques de la grâce que doit franchir le voyageur de la vie.

彼の嫌悪したのは束縛であり禁制だつた。彼の憧憬した国士は自由と創造と の世界だつた。彼は人間内性の欲望が無限の力を宿す事を信じてみた。彼は抑 圧に対する強烈なによつて人生の無限なを讃えてみる。自己実現に対する偉大 な抱負は技が生涯失はなかつた理想である。彼は之を制定するあらゆる法則を 生命の名によつて勇ましくも破壊した。自由と解放とは人生の旅人が通過すべ き唯一の祝福ある金門だつた。 
Yanagi n'a quasiment pas évolué dans sa conception du génie individuel. Toutefois, quand on compare avec les textes des années 1910-1912, on voit qu'il a acquis une vision plus mystique de l'individu. Ce qu'il perçoit chez les grands artistes, et Blake en particulier, c'est le singulier qui retrouve le tout. Comme il l'écrit en anglais à Bernard Leach dans une lettre datée du 8 novembre $1915^{15}$ :

Comme tu le sais déjà, je suis très intéressé par le mysticisme chrétien, en partie du fait de ma propre nature, en partie à travers l'étude de Blake. Je peine depuis longtemps sur le problème du dualisme, me battant avec les divisions de la vie - l'âme et le corps, le Paradis et l'Enfer, Dieu et l'homme, etc. Comment y échapper, s'en émanciper? - comment unifier ou organiser ces dualismes? - a été ma constante préoccupation. Quand j'ai été confronté pour la première fois à la pensée de Blake, dont le résultat s'exprime dans mon laborieux mais gratifiant travail sur cet étrange et grand génie de notre monde, une nouvelle vie a commencé.

14 On peut toutefois douter de l'originalité de cette "nouvelle vie ». Car, à la même époque précisément, Mushanokōji ou Kishida Ryūsei effectuaient un virage du même ordre. On peut aussi penser à Kurata Hyakuzō 倉田百三 qui publia en 1916 Shukke to sono deshi 出家とその弟子 (Le prêtre et ses disciples), une pièce de théâtre mettant en scène le moine Shinran 親鸞 qui connut un très grand succès ${ }^{16}$. La lecture de Blake, des penseurs chrétiens, mais aussi la découverte du zen ont certes aidé Yanagi dans son cheminement intellectuel et spirituel, mais, dans ce groupe très soudé que constituait Shirakaba à ses débuts, il y a une cohérence des trajectoires qui tend à transformer la quête d'absolu qui caractérise chacun d'entre eux en phénomène social.

\section{La matière à l'œuvre}

15 Jean-Jacques Origas disait de Yanagi qu'il a vécu « dans une relation constante à la terre $»^{17}$. Le mouvement des Arts populaires, qui met l'accent sur les œuvres du terroir, est la manifestation la plus connue de cet intérêt. On peut aussi interpréter sous cet angle et de façon symbolique son attachement au bouddhisme de la Terre pure (Jōdo shinshü), très fort dans la deuxième moitié de sa vie. Toutefois, on en voit des signes dès sa jeunesse, dans les années 1910 .

La passion de Yanagi pour la terre s'est d'abord manifestée à travers son amour de la céramique, un art qui engendre des objets aux formes diverses et aux usages variés à partir de la glaise. D'autant que les œuvres qu'affectionne Yanagi sont relativement peu décorées et laissent deviner au regard ou au toucher la matière qui les forme. Yanagi a par ailleurs noué des liens avec des potiers bien avant de lancer le mouvement des Arts populaires, à commencer par Tomimoto Kenkichi 富本憲吉 qu'il a connu dès 1912. Il suivit aussi de près à Abiko 我孫子 les progrès de Leach en la matière, l'aidant même à construire un four dans son jardin. Il commença enfin une petite collection de céramiques chinoises dès les années 1910, avant de l'élargir à la poterie coréenne de la dynastie Yi dans les années 1920, puis à tous les objets populaires. En s'installant à la campagne à partir de 1914, en suivant de près les travaux des potiers, en commençant à collectionner des céramiques, Yanagi était manifestement séduit par la figure de l'artiste démiurge.

On sait qu'une des images qui ont fait le plus rêver les artistes des années 1910 est l'image biblique de la création par Dieu du monde et de l'homme. Chez Yanagi, cela s'est notamment manifesté par une véritable fascination à l'égard de William Blake 
chez qui ce thème est omniprésent. La monographie que Yanagi publie en 1914 sur l'artiste anglais commence d'ailleurs par une évocation de l'une des plus célèbres œuvres de l'artiste anglais, The Ancient of Days (1797), reproduite dans le texte, où l'on voit Urizen - le Yaveh de Blake - créant le premier cercle de la Terre ${ }^{18}$ :

Le vieux dieu assis au centre du soleil, le corps recroquevillé, contemple de haut le ciel azuré. Il tend sa main gauche qu'il ouvre comme un compas, définissant ainsi les contours de la terre. Le dieu s'apprête à créer la terre primordiale de l'espace vide du chaos.

年老いた神が太陽の中央に座つて身を屈め乍ら遠く珵空を下に見おろしてる

る。彼は今左手を長く延べてコムパスの度を拡げ、地球の圈囲を定めてるる。

神は渾沌の虚空から太初の地を造らうとするのである。

Le thème biblique de la Création du monde (ou ses variantes comme celle inventée par Blake) est un motif qui s'observe chez plusieurs contemporains de Yanagi. Kishida Ryūsei a réalisé en 1914, année de la publication du Blake, une petite série de gravures où l'on voit Adam mordre la terre à pleines dents. Bien que sans lien apparent avec la Bible, on pourrait aussi citer plusieurs poèmes de Takamura Kōtarō 高村光太郎 dans Étapes (Dōtei 道程, 1914), où le motif de la terre est utilisé comme métaphore de l'éveil créateur. Sur un mode plus subversif, «La langue du diable » (Akuma no shita 悪魔の舌) de Murayama Kaita 村山槐多 est une nouvelle, là encore de 1914-1915, où le héros, un poète du nom de Kaneko, relate sa longue déchéance depuis le jour où il commencé à manger de la terre jusqu'à celui où il finit par dévorer le cadavre de son propre frère qu'il a assassiné sans l'avoir reconnu ${ }^{19}$.

En termes phénoménologiques, valoriser l'emploi de la terre, apprécier son contact, c'est affirmer l'importance de ce qui est malléable, visqueux, indéfinissable, par opposition à ce qui est dur, formel, clos. Autrement dit, cela revient à rejeter le primat de la représentation abstraite, de la vérité scientifique, de l'expérience reproductible. Toutefois, dans la logique de Yanagi au début de l'ère Taishō, seuls les génies, seules des formes supérieures de l'humanité sont capables de ne pas se laisser engloutir dans la matière brute et de lui donner vie. Tel est le sens de son intérêt pour Blake, mais aussi pour Rodin, Van Gogh ou encore Cézanne.

La terre est malgré tout un élément dangereux car elle porte en elle non seulement des promesses de création, mais aussi le rappel de la mort. Elle est l'origine mais aussi le futur de l'homme. L'artiste qui s'y confronte directement est toujours amené à osciller entre le sentiment de puissance qu'il y a à la dominer et l'impression qu'il est impossible de lui échapper. Yanagi en a évidemment conscience, comme Blake avant lui. Vu dans cette perspective, l'appel qu'il formulera à partir des années 1925-1926 en vue de dépasser la volonté d'art dans l'abandon au faire et l'exercice inconscient de la main est d'une certaine manière la conséquence d'une reddition de sa part. Ce n'est pas tant Yanagi qui a "découvert» les arts populaires et la puissance heuristique et anonyme de la terre, que la terre qui a soumis à sa force celui qui pensait pouvoir la dominer. Le chemin qui mena au mouvement Mingei est davantage celui d'une défaite que celui d'une révélation positive.

\section{Des images réenchantées}

21 La carrière universitaire de Yanagi est un aspect de sa vie étonnamment méconnu. C'est au printemps 1919 que Yanagi fut nommé professeur du département de philosophie de l'université privée Tōyō Daigaku 東洋大学 (Tōkyō) dont le fondateur et la figure de proue était Inoue Enryō 井上円了 (1858-1919). Ce dernier décéda en Mandchourie en 
juin de la même année. Les deux hommes n'ont donc pas pu se connaître étroitement, toutefois il est certain qu'Inoue a joué un rôle décisif dans la nomination de Yanagi ${ }^{20}$. D'une certaine manière, l'un fut le successeur de l'autre.

Ce rapprochement n'est pas sans intriguer. Inoue Enryō est connu pour avoir, durant des décennies, cherché à expliquer au peuple la nature véritable des phénomènes étranges et autres confusions de l'esprit. Homme d'un immense savoir, fervent bouddhiste et grand partisan de la cause nationale, il mit toute son énergie à exploiter les vertus d'une science positive et illuminatrice ${ }^{21}$. Par certains aspects, l'action de Yanagi s'inscrit dans le prolongement de celle d'Inoue. Tous deux avaient une grande puissance de travail, une capacité à synthétiser les connaissances modernes, un souci de parler au peuple. Toutefois, la fascination de Yanagi pour les objets et les images les distingue de façon nette. Alors qu'Inoue réclamait que les artistes missent leur talent au service du réel et de la vérité, Yanagi a toujours eu une relation aux images de l'ordre du magique.

Prenons l'exemple de son article de mars 1911 sur Renoir, où Yanagi décrit abondamment les œuvres du maître de Cagnes. Contrairement à ce qu'on pourrait attendre, il ne donne que peu de détails biographiques. En revanche, il décrit abondamment les tableaux et de façon précise. Ainsi, Lise (1867) et La Danseuse (Maihime en japonais), une œuvre de 1874 (non reproduite dans le texte) qu'il présente comme suit $^{22}$ :

Dans ce tableau, La Danseuse, le même blanc léger qui baignait la toile Lise, saisit plus encore les formes et souligne les chairs de la jeune fille. Les pans de son tutu bleu clair semblent se fondre presque totalement dans l'arrière-plan, le contour flou de ses formes, ses cheveux bruns, ses chaussons roses apparaissent comme autant de petites touches colorées; c'est une toile dont émane un effet indescriptible; dont l'impression générale est celle d'une richesse absolue des couleurs [...]

此「舞姫」の画では、かの"Lise"を包んだ淡い白色は更に形を納めて若い女 の肉体を強めて居る、浅藍色の沙の裾は殆ど背後に溶け込む様で、朧ろげな外 廓や裮色の鬞や桃色の鞉等は只一筆の色に触れた ゙けげあるが、其全体の印象 は色彩の豊富を以て満ちて居る、

Plus loin, comparant Renoir à Degas, Yanagi évoque ${ }^{23}$ :

Ces carmins clairs comme ceux de pêches mûres, ces rouges profonds comme des tomates éclatées, les bleus de jade qui brillent dans le ciel du Sud, toutes ces couleurs qu'il nous met sous les yeux et pour lesquelles il excelle [...]

彼は勝ち誇れる色彩を目のあたりに示した、熟せる桃の如き薄紌、裂けたる トマトーの如き深い赤、南の空の光れる碧（後略）

Quand on repense aux goûts de Yanagi tels qu'ils s'exprimeront plus tard dans le mouvement Mingei, on ne peut être que surpris de la sensualité, du lyrisme qui s'expriment ici. Dans un style à la fois précis et généreux, Yanagi tente de cerner des images qui toujours résistent à la description. Tel tableau reste "plein de mystère " (fushigi ni michita), tel autre "fait penser à un village féerique " (yōsei no sato o omowaseru) ${ }^{24}$. On est loin du bouddhisme positiviste d'Inoue.

Ceci est encore plus évident dans «La nouvelle science» ("Atarashiki kagaku»), un article de 1910 où Yanagi présente, d'après des sources scientifiques occidentales ${ }^{25}$, divers récits d'apparitions, de maisons hantées et autres manifestations étranges. Alors que devant de tels phénomènes, Inoue opposait les apparences à la vérité, Yanagi voit dans la science et les arts un révélateur nouveau de l'« âme du grand tout; du sage silence ; de la beauté universelle à laquelle chaque partie et particule est uniformément reliée ${ }^{26}$. La vérité est pour lui présente à même les apparences. 
gi reproduit dans son texte plusieurs clichés de «spectres " que l'opérateur a réussi à saisir par une technique spéciale, ou qui se sont invités dans l'image à son insu. Il en conclut ${ }^{27}$ :

Si l'on considère ces différents faits comme exacts, nous devons admettre que nous percevons grâce à la sensibilité de la photographie des choses dont nous ne pouvons avoir connaissance avec nos propres yeux, et qu'il est en outre bien possible que flotte dans l'éther une multitude d'esprits.

若しかっる事実を真なりとすれば、吾人が肉眼によつて認識し能はざるものが 鋭敏なる写真に感じるのである、更に推測すれば此大気中には、幾多の精霊が 彷得へると見る可きである。

Bien que Yanagi aborde tous ces phénomènes avec rigueur et méthode, on sent qu'il est très sensible à l'idée que, derrière les limites du regard, le monde est un bouillonnement de forces mystérieuses et interagissantes ; une vie anime les objets pour qui a les moyens techniques, intellectuels ou spirituels pour le percevoir.

Au début des années 1910, Yanagi n'avait encore jamais quitté le Japon. Sachant qu'il n'y avait à cette époque aucune collection publique d'art moderne, il n'avait à sa disposition pour connaître les œuvres occidentales que des livres et des séries de cartes postales importés. Donc des reproductions photographiques, des objets de même nature que ce qui lui servait à reconnaître l'existence des spectres. La plupart du temps, ces reproductions étaient des tirages en noir et blanc, même lorsqu'il s'agissait de livres occidentaux comme la célèbre série des éditions Piper \& Co de Munich sur Cézanne (1910), Renoir (1911) ou Van Gogh (1911) qui ne comprend aucune planche en couleurs. Yanagi était alors l'un des collectionneurs d'images et d'albums illustrés les plus acharnés, chez qui se retrouvaient pour les admirer de nombreux artistes et écrivains de l'époque, comme Kishida Ryūsei, Kimura Shōhachi 木村荘八 ${ }^{28}$ ou Mushanokōji Saneatsu ${ }^{29}$.

29 Cette observation nous conduit à souligner un phénomène bien connu, à savoir que les reproductions ont joué une part décisive dans l'aventure de l'art moderne au Japon. Kinoshita Nagahiro a été l'un des premiers à analyser ce phénomène ${ }^{30}$ :

Ceux qui n'avaient vu que des reproductions restaient intellectuellement actifs, tandis que ceux qui avaient vu les euvres en vrai s'enfermaient dans un morne silence excluant la réflexion. (Cela signifie que l'on ne peut considérer comme supérieure l'expérience des ceuvres originales.)

複製しか見ない者は理屈が多くなり、本物を見た者はそこに理屈にならない貴 重な経娩を抱えて寡黙になるといい切ってしまう【ということは、本物を経騒 していることの優位性を認める】わけにはいかない。

Le caractère incomplet de la reproduction a stimulé l'imagination et la créativité des artistes restés dans l'archipel, tandis que la révélation brutale des originaux a pu au contraire assécher le regard de ceux ayant fait le voyage jusqu'en Europe. Récemment, Tsuchida Maki 土田眞紀 a poursuivi le travail de Kinoshita. Dans un article consacré à Yanagi et à l'ensemble des membres du groupe Shirakaba, elle décrit l'enthousiasme qui les a saisis face aux œuvres occidentales. Ce qui l'amène à la conclusion suivante ${ }^{31}$ :

Si on compare leur attitude face aux reproductions et leur attitude face aux " originaux » de Rodin ${ }^{32}$, il semblerait qu'il n'y ait eu pour eux qu'une différence de degré, et pas un changement de nature. [...] On peut penser que les reproductions étaient davantage pour eux que des substituts aux originaux; elles possédaient une forme d' "exactitude", dont l'expérience était clairement pour ceux qui la faisaient une expérience du hic et nunc et de l'unique rencontre. 


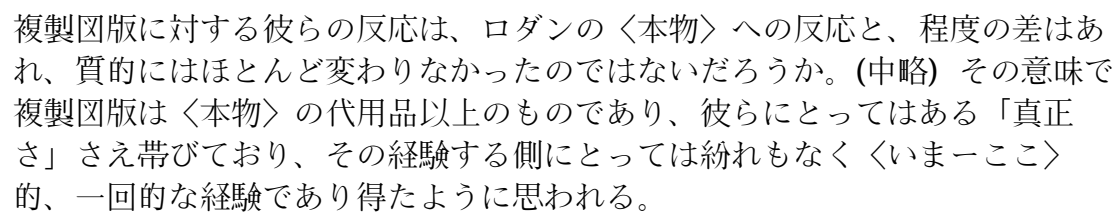

31 Par sa nature délocalisée, par son étrange matérialité, parce qu'enfin elle induit une attente, la reproduction semble avoir en effet renforcé chez nombre d'artistes modernes du Japon la perception de l'image en général comme puissance irradiante.

Cette sensibilité exacerbée est loin toutefois de se limiter aux reproductions photographiques. Tous les objets pouvaient avoir aux yeux de Yanagi un caractère enchanté débordant du cadre de leur fonction ou de leur signification. Il faut garder ce point à l'esprit lorsqu'on aborde son travail sur les arts populaires. Son rapport aux objets usuels comme la céramique coréenne de la dynastie Yi est incompréhensible hors de cette perspective.

\section{Découverte individuelle du nous ou crise collective du moi?}

Le discours et les priorités de Yanagi en matière de goût ont évolué de façon sensible autour de 1920. C'est en 1916 qu'il a découvert l'art coréen, au cours d'un voyage dans la péninsule. Trois ans plus tard, en juillet 1919, il publie dans Shirakaba quelques reproductions d'art bouddhique japonais, ce qui est une première pour la revue. En février 1920, il rédige un premier article sur la sculpture coréenne, accompagné là encore de plusieurs illustrations. C'est un tournant auquel on rattache volontiers l'émergence de sa théorie des arts populaires.

Au cours de son voyage en Corée, Yanagi a été touché par la condition des populations sous domination japonaise. En 1920, dans un article intitulé "Lettre à mes amis coréens » («Chōsen no tomo ni okuru sho »朝鮮の友に贈る書), il écrit ${ }^{33}$ :

En ce moment, j'espère de tout mon coeur qu'adviendra un jour où sera corrigée la relation
anormale qui existe entre nos deux pays. La Corée, qui est en vérité un pays frère du Japon,
ne doit pas devenir son esclave. Ceci n'est pas seulement le déshonneur de la Corée, c'est
d'abord une honte absolue pour le Japon.
私は今、二つの国にある不自然な関係が正される日の来ることを、切に希って
いる。まさに日本にとっての兄弟である朝鮮は、日本の奴隷であってはなら
ぬ。それは朝鮮の不名誉であるよりも、日本にとっての恥辱の恥辱である。

35 À partir de cette date, Yanagi a clairement tendance à considérer l'art d'un point de vue non plus individuel, mais national et collectif. Il ne publie plus de textes monographiques comme il l'avait fait sur Blake, Renoir ou Rodin, mais s'intéresse à des époques, des genres, des styles: « l'art Song », « l'art de la dynastie Yi », « la céramique coréenne ", «l'art japonais ». L'émergence de sa théorie des arts populaires s'inscrit dans ce cadre conceptuel nouveau qui valorise les productions de groupe et les catégories générales.

Cette nouvelle orientation l'amène à progressivement rejeter le mérite de la création individuelle, comme on le voit dans Le beau et l'artisanat (Bi to kōgei), qu'on peut considérer comme le premier texte programmatique complet du mouvement Mingei ${ }^{34}$ :

Pourquoi la beauté apparaît-elle de façon beaucoup plus forte dans les "objets ordinaires " que dans les " objets raffinés" ? Cela tient d'abord à une différence de disposition d'esprit au moment de la création. Au fait que l'absence d'intention présente dans les premières se situe 
dans des territoires plus purs que l'intentionnalité des secondes. Au fait que le détachement implique quelque chose de bien plus profond que la conscience. Au fait que l'oubli de soi est une base plus solide que la maîtrise de soi. Au fait que l'anonymat fournit un environnement plus serein que la signature [de son cuvre]. Au fait que la nécessité est une bien meilleure garantie de la beauté que l'acte volontaire.

なぜ「上手物」より却て「下手物」にかくも豊かな美が現れてくるか。それは

一つに作る折の心の状態の差異によると云はねばなりません。前者の有想より

も後者の無想が、より清い境地にあるからです。意識よりも無心の方が、更に

深いものを含むからです。主我の念よりも忘我の方が、より深い基礎となるか

らです。在銘よりも無銘の方が、より安らかな環境にあるからです。作為より

も必然が、一層厚く美を保証するからです。

Alors que, dans les textes des années 1910, l'individu était le centre de tout, que rien ne semblait pouvoir se faire en dehors de la subjectivité de l'artiste, à partir des années 1920, la subjectivité est rejetée, l'artiste authentique devenant celui qui, dans la réalisation de son geste, oublie toute volonté de faire art. Ce changement à quelques années d'intervalle a en soi le mérite de souligner très nettement l'historicité du discours bouddhisant de Yanagi visant, comme dans la dernière citation, à valoriser un rapport détaché de l'artiste à son œuvre, détachement dû à l'automaticité du geste et à l'absence de prétention à laisser une trace personnelle.

On parvient à une conclusion similaire quand on regarde l'évolution du style de l'écrivain. Au cours des années 1910, sa langue est précise, puissante, mais elle est aussi très dynamique. Or, à partir du début de l'ère Shōwa, il adopte une écriture simple, répétitive, presque ratiocinante. Alors qu'il était capable de lyrisme dans ses descriptions d'œuvres, comme on l'a aperçu dans son article sur Renoir, sa langue tend à s'assécher et se dépersonnaliser. Nombreux sont les textes des années 1930 à 1950 qui ne sont que des successions de formules prévisibles et faciles à retenir, avec des effets lourds de questions et réponses. Il ne s'adresse plus à ses pairs comme à l'époque de Shirakaba, mais à un " peuple » moyen et anonyme.

Toutefois le mouvement de retour au peuple opéré par Yanagi est loin d'être solitaire. Par bien des aspects, il ne fit que réagir à la nouvelle donne du cercle intellectuel dans lequel il évoluait. Après huit ou neuf ans où tous les membres du mouvement Shirakaba avaient proclamé à l'unisson la force de leur moi, chacun esquissa un mouvement de côté et entreprit, alors que la Révolution russe et la fin de la Première Guerre mondiale bouleversaient les consciences, de repenser l'individu par rapport à un collectif culturel, social ou national. C'est le cas de Mushanokōji et de Kurata qui tentèrent à partir de 1918 une aventure agrarienne en fondant leur Nouveau village; d'Arishima Takeo qui se rapprocha des mouvements socialistes et participa en 1921 au lancement de la revue Tanemaku hito 種蒔く人 (Les semeurs) ${ }^{35}$; d'Abe Jirō 阿部次郎 et de Watsuji Tetsurō 和辻哲郎 qui proposèrent une redécouverte du patrimoine littéraire et architectural national ${ }^{36}$; de Kishida Ryūsei et de Kimura Shōhachi qui se mirent à collectionner et étudier l'art populaire d'Edo. La création du mouvement des Arts populaires par Yanagi peut donc se lire comme une adaptation à un mouvement plus général au sein des intellectuels et artistes de sa génération. L'évolution de sa pensée et de son style est par bien des aspects une série de reprises d'appui, une mise à niveau de son discours en réaction, d'une part, aux grandes évolutions historiques, d'autre part, à la combustion de ses propres matériaux d'étude. suivre Mushanokōji dans son retour aux champs et à la communauté villageoise. Il n'a 
pas non plus eu la noire lucidité d'Arishima Takeo qui, quelques mois avant de se suicider, écrivait dans une forme de manifeste-testament ${ }^{37}$ :

On peut toujours être un grand savant, un penseur, un meneur d'hommes ou un chef, quand on n'est pas prolétaire, vouloir aider en quoi que ce soit le prolétariat, est clairement ridicule. Le prolétariat ne saurait être que perturbé par les vains efforts de ces gens.

どんな偉い学者であれ、思想家であ机、運動家であ机、頭領であ机、第四階 級な労㗢者たることなしに、第四階級に何者をか寄与すると思つたら、それは 明らかに筚上沙汰である。第四階級はその人たちのむだな努力によつてかき乱 されるのほかはあるまい.

41 Yanagi, tout en défendant les arts populaires et le génie de celui qui crée de façon spontanée et anonyme des objets usuels, est resté dans une posture d'intellectuel influent et de collectionneur aisé. Après tout, comparée au fiasco de Mushanokōji qui quitta son Nouveau village en 1926 par souci de donner une bonne éducation à ses filles et à la fin tragique d'Arishima qui, accompagné de sa maîtresse, mit un terme à ses jours à Karuizawa le 9 juin 1923, l'attitude de Yanagi a le mérite d'une certaine solidité. Il y a néanmoins, dans le confort matériel et moral de son parcours, une lourdeur qui se répercute sur son style comme une vengeance de l'histoire.

\section{Conclusion}

Contrairement à ce que dessine l'exposition organisée au Musée du quai Branly, il n'y a pas continuité entre les œuvres du patrimoine populaire ancien et des œuvres de design contemporain. Le " nous » que semblent partager les céramiques et les tissus de l'époque d'Edo rassemblés par Yanagi et les œuvres de son fils Yanagi Sōri 柳宗理 (1915-) n'est pas du même ordre. Le « nous » du mouvement des Arts populaires est un nous de crise, reconstruit suite à un épuisement du «moi » et à la pression des événements historiques à l'étranger. Le Mingei n'est pas le chaînon qui unit la profondeur de la culture japonaise - sa part de mystère, de magie - à sa face moderne et efficace. Pour le dire autrement, le mouvement initié par Yanagi à la fin des années 1920, par bien des aspects, n'est que l'un des multiples rejetons du groupe Shirakaba. Du reste, l'historiographie japonaise ne s'y trompe pas, Shirakaba étant en définitive bien plus présent dans les musées publics et les manuels d'histoire que les œuvres du Mingei.

Si l'on avait voulu sortir d'un récit à la fois héroïque et exotique de l'action de Yanagi, il aurait fallu commencer par présenter de façon critique les multiples éléments qui permettent de la situer dans la durée, ce qui n'était pas le cas de l'exposition du Musée du quai Branly, loin s'en faut. Certes, on peut admettre que certaines manifestations organisées sur l'art japonais misent sur une présentation formelle et décontextualisée afin de séduire et de marquer un public nouveau. Toutefois, il s'agit souvent d'une facilité, voire d'une certaine paresse. On n'aurait que mieux apprécié les œuvres si on avait pu comprendre et sentir, au-delà du fait que Yanagi est le lien qui les unit, tout ce qu'elles comprennent de magie et d'incantation, de fêlure et de renoncement. 


\section{NOTES}

1. Élisabeth Frolet, Yanagi Sōetsu ou les éléments d'une renaissance artistique japonaise, Paris, Publications de la Sorbonne, 1986 ; Yanagi Sōetsu (adapté par Bernard Leach), Artisan et inconnu, Paris, L'Asiathèque, 1992. En anglais voir notamment Bernard Leach, A Potter in Japan 1952-1954, London, Faber \& Faber, 1960.

2. On trouvera de petits développements sur Shirakaba et son rôle dans l'histoire de l'art dans : Asano Tōru, « La peinture à l'époque Taishō », in Japon des avant-gardes, Paris, Éditions du Centre Pompidou, 1986, p. 51-52 ; Michael Lucken, L'Art du Japon au vingtième siècle, Paris, Hermann, 2001, p. 45-48. En japonais, voir, parmi les publications les plus récentes, le catalogue de l'exposition Shirakaba-ha no ai shita bijutsu, édité par Furutani Yoshiyuki, Ōsaka, Yomiuri shinbun, 2009.

3. Yuko Kikuchi, "The Myth of Yanagi's Originality: The Formation of Mingei Theory and its Social and Historical Context", Journal of Design History, ${ }^{\circ}$ 7, Oxford University Press, 1994, pp. 247-266; Yuko Kikuchi, “A Japanese William Morris: Yanagi Sōetsu and Mingei Theory”, Journal of William Morris Studies, $\mathrm{n}^{\circ}$ 12-2, 1997, pp. 39-45.

4. Nagayo Yoshirō (1888-1961), écrivain et dramaturge, ami d'enfance de Yanagi, a rejoint Shirakaba en 1911.

5. Bernard Leach (1887-1979), qui résida longuement au Japon entre 1909 et 1920, réalisa les couvertures de Shirakaba tout au long de l'année 1913 et ponctuellement en 1917 et 1918.

6. Celui-ci fut inauguré par Mushanokōji Saneatsu en 1918, dans une boucle de la rivière Omaru, à Hinata (Kijō, Koyu-gun), dans le département de Miyazaki, au sud-est de Kyūshū. Le village était géré de façon égalitaire par l'ensemble des membres. Mushanokōji demeura sur place jusqu'en 1926, date à laquelle il s'installa à Nara. Une petite communauté existe toujours à l'heure actuelle. 7. L'exposition "Manet and the Post-impressionists" fut organisée par Roger Fry (1866-1934) à Londres dans les Grafton Galleries en novembre 1910. Elle fit découvrir au monde anglo-saxon et au Japon les œuvres de Cézanne, Van Gogh, Gauguin ou encore Matisse. L'appellation “postimpressionism" est souvent attribuée à Fry.

8. Kinoshita Nagahiro, Shisō-shi toshite no Gohho. Fukusei juyō to sōzōryoku, Tōkyō, Gakugei shorin, 1992, p. 53 et suiv.

9. Yanagi Sōetsu, « Kakumei no gaka », Shirakaba, vol. 3-1, janvier 1912 ; Yanagi Sōetsu zenshū (plus loin YSZ), vol. 1, p. 563-564.

10. Traduction publiée sous le titre Tsaratusutora ツァラトゥストラ (Tōkyō, Shinchōsha, janvier 1911). Ikuta Chōkō (1882-1936), écrivain et traducteur spécialiste de Nietzsche, était un proche du mouvement Shirakaba ; il a publié à plusieurs reprises dans la revue.

11. Sur Kishida Ryūsei (1891-1929), voir, Michael Lucken, L'Art du Japon au vingtième siècle, Paris, Hermann, 2001, p. 59-78, et «Une esthétique de la réplication ou comment les fantômes sont à l'œuvre. La peinture de Kishida Ryūsei », Arts asiatiques, tome 64, Musée Guimet / EFEO, 2009, p. 79-94.

12. La Société du Fusain (Hyūzan-kai ヒューザン会 puis Fyūzan-kai) comprenait une quinzaine d'artistes, essentiellement des peintres occidentalisants, dont Saitō Yori, Yorozu Tetsugorō et Kishida Ryūsei. Elle organisa en octobre-novembre 1912 et mars 1913 deux expositions qui firent date dans l'histoire de l'expressionnisme et des avant-gardes en général au Japon.

13. Kishida Ryūsei, "Jiko no geijutsu», Yomiuri shinbun, 17 octobre 1912, p. 5 ; Kishida Ryūsei zenshū, vol. 1, p. 39-40.

14. Yanagi Sōetsu, Wiriamu Burēku, Tōkyō, Rakuyōdō, 1914 ; YSZ, vol. 4, p. 19.

15. In Bernard Leach, Beyond East and West: Memoirs, Portraits and Essays, London, Faber \& Faber, 1978 (1985), p. 79-80. 
16. Kurata Hyakuzō (1891-1943), écrivain et dramaturge du mouvement Shirakaba. Sa pièce Shukke to sono deshi fut publiée en feuilleton en 1916, avant d'être reprise en volume par les éditions Iwanami (1917). Traduite en anglais dès 1922, elle fut remarquée par Romain Rolland qui rédigea la préface de la traduction française (Le prêtre et ses disciples, Kuni Matsuo et Émile Steinilber-Oberlin, Paris, Rieder, 1932).

17. Jean-Jacques Origas, "Yanagi Sōetsu: les mots, les images et la terre", in La Lampe d'Akutagawa. Essais sur la littérature japonaise moderne, Paris, Les Belles Lettres, coll. « Japon », 2008, p. 326.

18. Yanagi Sōetsu, Wiriamu Burēku, op. cit., YSZ, vol. 4, p. 15.

19. Cf. Takamura Kōtarō, Takamura Kōtarō shishū, Tōkyō, Shinchōsha, 1950 (1990); Muyarama Kaita, Muyarama Kaita zenshū, Tōkyō, Yayoi shobō, 1997.

20. Yanagi était par ailleurs le neveu par alliance de Kanō Jigorō 嘉納治五郎, le fondateur du jūdō, qui connaissait bien Inoue Enryō.

21. Cf. Inoue Enryō senshū, 25 vol., Tōkyō, Tōyō daigaku, 1987-2004. Sur Inoue Enryō, voir en anglais : Gerald Figal, Civilization and Monsters: Spirits of Modernity in Meiji Japan, Durham / London, Duke University Press, 1999, passim.

22. Yanagi Sōetsu, «Runoā to sono ippa », Shirakaba, vol. 2-3, Tōkyō, mars 1911, p. 3 ; YSZ, vol. 1 , p. 493.

23. Ibid., p. 5 ; YSZ, vol. 1, p. 495-496.

24. Ibid., p. 6 ; YSZ, vol. 1, p. 496-497.

25. Yanagi s'appuie particulièrement sur Cesare Lombroso, After Death -What?, Boston, Small Maynard \& Co, 1909; Oliver Lodge, The Survival of Man, London, Methuen, 1909 ; Camille Flammarion, Les Forces naturelles inconnues, Paris, Flammarion, 1907 (Yanagi utilise la traduction anglaise). On notera que ces deux derniers livres figuraient à la même époque dans la bibliothèque de Sōseki, signe de leur impact au Japon.

26. Traduction d'un passage d'Emerson cité par Yanagi en anglais en épigraphe de son article. Cf. Yanagi Sōetsu, «Atarashiki kagaku», Shirakaba, vol.1-7, Tōkyō, octobre 1910, p.16-18; YSZ, vol. 1, p. 35.

27. Ibid., p. 18 ; YSZ, vol. 1, p. 46.

28. Kimura Shōhachi (1893-1958), peintre occidentalisant. Membre du groupe Sōdo-sha (Société de l'Herbe et de la terre) et ami de Kishida Ryūsei, il a eu une importante activité critique et a publié à plusieurs reprises dans Shirakaba. Comme Kishida Ryūsei, il s'intéresse à partir de 1920 à l'ukiyo-e et au kabuki.

29. Cf. "Omoide oyobi kondo no tenrankai ni sai shite », Kishida Ryūsei zenshū, vol. 2, Tōkyō, Iwanami shoten, 1979, p. 235 ; Kimura Shōhachi nikki, Nikkō, Kosugi Hōan kinen Nikkō bijutsukan, 2003, p. 190.

30. Kinoshita Nagahiro, Shisō-shi toshite no Gohho, op. cit., p. 51.

31. Tsuchida Maki, "Yanagi Sōetsu to "kindai bijutsushi": "miru" to iu jissen ", in Taishō-ki bijutsu tenrankai no kenkyū, Tōkyō, Tōkyō bunkazai kenkyūjo, 2005, p. 565 et 567. L'auteur s'inspirant ouvertement de Benjamin, nous reprenons dans notre traduction en français la terminologie benjaminienne.

32. Référence aux trois sculptures envoyées par Rodin fin 1911 aux membres de Shirakaba en remerciement du lot d'estampes ukiyo-e que ceux-ci lui avaient fait parvenir quelques mois auparavant. Voir notamment François Blanchetière, « Don et contre-don : Rodin et le groupe Shirakaba ", in Rodin. Le rêve japonais, Paris, Musée Rodin / Flammarion, 2007, p. 195-219.

33. Ce texte publié dans la revue Kaizô sera repris en 1922 dans son livre Chōsen to sono geijutsu ( $L a$ Corée et ses arts), Tōkyō, Sōbunkaku ; Yanagi Sōetsu, Mingei yonjū-nen, Tōkyō, Iwanami shoten, coll. « Iwanami bunko », 1984, p. 26. 
34. Yanagi Sōetsu, Bi to kōgei, Tōkyō, Kensetsusha, 1934 ; YSZ, vol. 8, p. 296. Ce texte fut publié pour la première fois en juin 1928 dans la revue Bungei shunjū sous le titre « Nani o getemono kara manabiuru ka ».

35. Sur Tanemaku hito, voir Jean-Jacques Tschudin, Les Semeurs - Tanemaku hito : la première revue de littérature prolétarienne japonaise, Paris, L'Asiathèque, 1979.

36. Abe Jirō (1883-1959) et Watsuji Tetsurō (1889-1960), philosophes. Au cours de l'ère Taishō, ils ont consacré une bonne part de leur énergie à la réflexion esthétique. Les deux, mais surtout le premier, étaient étroitement liés au mouvement Shirakaba. En 1920, ils participèrent, entre autres, à la fondation d'une Société d'étude sur [le poète] Bashō (Bashō kenkyūkai).

37. Arishima Takeo, «Sengen hitotsu », Kaizō, janvier 1922; Arishima Takeo zenshū, vol. 9, Tōkyō, Chikuma shobō, 1981, p. 3.

\section{RÉSUMÉS}

Durant les années 1910, Yanagi Sōetsu s'investit dans la revue Shirakaba. Bien avant le mingei, deux questions sous-tendent son travail : l'individu créateur et l'œuvre d'art.

During the 1910s, Yanagi Sōetsu was involved in developing the Shirakaba review. Several years before the birth of mingei, he was interested in 2 themes: the creator and the artwork.

\section{INDEX}

Thèmes : histoire de l'art, littérature

キーワード : geijutsu 芸術, geijutsu sakuhin 芸術作品, bungei zasshi 文芸雑誌, Shirakaba 白樺, Yanagi Sōetsu 柳宗悦 (1889-1961), bijutsushi 美術史, bungaku 文学, Taishō jidai 大正時代 (1912-1923), Shōwa jidai 昭和時代 (1923-1945), Shōwa jidai 昭和時代 (1945-1989)

Mots-clés : création artistique, œuvre d'art, revues littéraires, Shirakaba, Yanagi Sōetsu (1889-1961)

Keywords : Artistic Creation, Literature, Literature - Periodicals, Shirakaba, Yanagi Sōetsu (1889-1961)

Index chronologique : Shōwa (1923-1945), Shōwa (1945-1989) 